\title{
Parodies de la justice dans le théâtre de John Day
}

Jean-Pierre Villquin

Marie-Thérèse Jones-Davies (éd.)

\section{OpenEdition}

\section{Journals}

Édition électronique

URL : http://journals.openedition.org/shakespeare/118

DOI : $10.4000 /$ shakespeare. 118

ISSN : 2271-6424

Éditeur

Société Française Shakespeare

\section{Édition imprimée}

Date de publication : 1 novembre 1980

Pagination : 51-60

Référence électronique

Jean-Pierre Villquin, "Parodies de la justice dans le théâtre de John Day », Actes des congrès de la

Société française Shakespeare [En ligne], 2 | 1980, mis en ligne le 01 novembre 2007, consulté le 29 avril 2019. URL : http://journals.openedition.org/shakespeare/118; DOI : 10.4000/shakespeare.118 


\section{SOCIÉTÉ FRANC̣AISE SHAKESPEARE}

\section{ACTES DU CONGRĖS 1980}

DIRECTEUR DE LA PUBLICATION

M.T. Jones - Davies

JEAN TOUZOT Libraire - Editeur 38 , rue Saint-Sulpice 75278 PARIS CEDEX 061981 


\section{PARODIES DE LA JUSTICE DANS LE THEATRE DE JOHN DAY}

Un jugement équitable, un châtiment mérité, une réparation nécessaire apportent une satisfaction, une purgation qui participent de la «catharsis» aristotélicienne. Au théâtre comme dans un procès public, il y a d'un côté les spectateurs, les juges, le jury et de l'autre les acteurs, plaignants et accusés qui jouent leur rôle, sorte de pièce dans la pièce, avec les mêmes rites, la même rhétorique, les même effets. Un jugement est l'aboutissement d'une action judiciaire comme un dénouement est l'aboutissement du drame. Les tragédies de Sackville, de Marlowe, de Kyd, de Shakespeare se sont nourries de l'idée de justice ou d'injustice et même si les voies qu'elles empruntent sont douteuses, même si les conclusions qu'elles proposent sont ambiguës, on y trouve rarement de véritable parodie. En effet, la parodie, ce chant parallèle, a longtemps épargné la justice pour ne toucher que ses serviteurs et les aigrefins de tout poil qui sont ses clients attitrés. Cette mine inépuisable est le vrai domaine de la comédie. Le comique naît alors de deux représentations qui tour à tour s'imbriquent et se répondent : la vraie, celle du théâtre et celle de la justice. Dans cet univers où la fiction devient réalité, la parodie oscille entre deux forces, l'une inflationniste qui tend au burlesque, l'autre déflationniste qui tend à la dérision. Tout ce qui gravite autour des gens de basoche se prête à ces jeux et à ces artifices. Les comédies et les mélodrames élisabéthains qui s'inspirent des affaires criminelles sont innombrables. John Day qui écrivait entre 1598 et 1635 environ exploita cette veine et les quelques œuvres qui ont survécu: ${ }^{1}$ reflètent à la fois la réalité de la justice, des juges et des prisons de Londres, mais offrent aussi une image déformée, sorte d'anamorphose qui démasque les errements de la justice et les impostures des gens de loi.

Toutes les pièces de John Day, à des degrés divers, apparaissent comme des procès dans leurs thèmes et dans leur structure. Pour suivre le dramaturge sur cette voie parallèle et souvent oblique qu'est la parodie, il nous faut un système de référence : The Parliament of Bees, allégorie en marge du théâtre, nous le donne. Dans ce parlement qui 
emprunte tout au rituel judiciaire, Prorex fait appeler les plaignants et, assisté du procureur et de ses baillis, les entend exposer leurs griefs. Il juge selon la loi et l'esprit de la justice dont le but, comme le proclame la devise qui accompagne le dessin de Bruegel représentant cette vertu cardinale, est d'amender ceux qui sont châtiés, de maintenir les autres dans le droit chemin et de lutter contre le crime afin d'assurer à l'homme une vie plus sûre ${ }^{2}$. La sécurité et l'équilibre de la république sont un souci permanent de ce monde en miniature et l'un des «caractères», l'abeille hospitalière, se propose de créer une institution afin d'enseigner l'usage des lois et d'assurer aux pauvres l'assistance judiciaire. Dans cette communauté mi-réelle mi-utopique, les bons sont récompensés et les méchants soumis à la «loi russe» ou loi du Talion. Justice distributive justice commutative s'allient pour faire régner l'ordre, et dans le dernier tableau, on voit Obéron, juge suprême, siéger dans la Chambre Etoilée. The Parliament of Bees n'est ni une tribune ni un exutoire, mais comme le déclare Day lui-même, c'est une «description allégorique des actions des hommes de notre temps, des bons comme des mauvais». Cette vision positive, objective de la justice servira ici de contrepoint à la parodie.

En effet, la justice n'est pas toujours représentée d'une manière aussi sereine qu'au royaume des abeilles. Dans Law Tricks, dont le titre résume le thème, toute l'intrigue se noue autour d'une sordide affaire domestique, ce serait une simple parodie de l'amour conjugal si le mari, Lurdo, n'était aussi homme de loi. Celui-ci soudoye les témoins, refuse de payer une pension, tente de faire assassiner sa future ex-femme, mais veille surtout à ce que toutes ses actions aient l'apparence de la légalité. Il sait tricher avec la loi, mot qui revient comme un leitmotiv à chaque réplique, la loi est la clé qui paradoxalement permet l'impunité à celui qui sait en user et en abuser. Au dénouement, pour sauver les apparences de la morale, le mauvais juge est jugé à son tour et avec l'Epilogue on s'étonne que des événements si tragiques tournent à la comédie, mais plus qu'un sacrifice aux conventions du genre, c'est une parodie : loi et justice apparaissent alors comme deux notions contradictoires.

Les grands schémas de la justice politique dans lesquels s'inscrivent les drames historiques, ceux de Shakespeare en particulier, sont traités sur un mode mineur. Si on 
prend l'exemple de The blind Beggar of Bednal Green, les titres, les hautes fonctions cachent de sinistres conspirateurs: cardinaux et grands seigneurs se conduisent en bandits, un noble barbon abuse de sa position de tuteur, le frère dépouille le frère, l'orgueil, la luxure, le goût du'lucre se substituent à l'honneur, à la passion, aux grandes ambitions. Au dénouement, le régent ne réussit à rétablir la «paix du roi» que dans un duel judiciaire qui s'achève en farce. La justice est donc bafouée ou absente, et les victimes honteuses. Momford, comme un Lear dépouillé de sa grandeur tragique doit se déguiser et supporter l'opprobre et les tracasseries qui s'attachent à la condition de mendiant et de vagabond. Les affaires de l'état perdent leur lustre et leur dignité car elles se réduisent à des intérêts privés. La juxtaposition des intrigues crée la confusion des valeurs, Momford, le baron déshonoré et spoilié et le béjaune de Tom Strowd qui se fait voler son manteau en appellent tous les deux à la même justice, mais le caractère dérisoire de cette dernière cause finit par ternir la première. Ce manque de discernement dans le degré d'importance des affaires qui relèvent de la justice est à son comble dans The Travels of The Three English Brothers. Les atteintes flagrantes au droit international, le mépris des libertés individuelles, les crimes les plus odieux ou les minimes égratignures faites à l'honneur chatouilleux des héros sont la source d'innombrables palabres procédurières. Les Shirley, ces ambassadeurs marginaux, marchands de canons et aventuriers, passent leur temps à être accusés, jugés et condamnés pour espionnage, pour piraterie ou seulement parce qu'ils sont chrétiens. Chantage, rançons, assassinats, emprisonnements s'accumulent à un rythme incroyable et tout ce qui touche à la justice ne semble être là que comme unique ressort d'une intrigue aussi itinérante que décousue.

John Day nous donne presque toujours une image négative de la justice, justice de théâtre certes, mais néanmoins reflet d'une réalité. Le public de Blackfriars était le même que celui qui allait voir pendre et écarteler les condamnés ou allait voir les fous et les prisonniers comme il allait voir les montreurs d'ours ou de singes ${ }^{3}$. Que la scène se déroule à Moscou, à Venise, à Rome ou en Arcadie, c'est toujours un Londres connu des spectateurs qu'évoque le 
dramaturge. C'est ainsi que Canbee, le coupeur de bourses, dépeint en quelques mots une scène quotidienne : «en passant par Allgate ce matin, j'ai vu les échevins et les baillis en route pour Newgate, le charpentier transportait le gibet à Bednal Green ${ }^{4}$. Le spectacle de la justice est partout présent. Que ce soit en filigrane ou avec un réalisme cru, les différentes instances judiciaires sont évoquées, depuis le juge de paix grand amateur de vin des Canaries, toujours à l'affût de la pratique, ${ }^{5}$ jusqu'au président du Banc du Roi, dernier recours avant le prince. Les allusions à la nécessaire délégation des pouvoirs, à la multiplicité des procédures, la référence constante aux actes de justice : mandats, placets, exploits, requêtes, écrou...etc. montrent à quel point la chicane faisait partie de la vie de chaque jour. Qu'ils soient magistrats au service du roi ou juristes au service de leurs clients, parfois les deux, et ce n'est pas là un des moindres aspects de la parodie, les gens de loi ont toujours mauvaise réputation. Le mariage d'un jeune juriste avec la vieille dame Conscience est un rêve ou serait un miracle ${ }^{6}$. Derrière le terme générique de «lawyer» qu'on retrouve dans toutes les pièces, se cache la cohorte des avoués, notaires, huissiers, suivis de la troupe des clercs qui ne pensent qu'à imiter leur maître dans l'art de la rapine. Tous sont des parasites, comme Dametas, des «champignons inutiles» qui pullulent et croissent aux dépens de la justice et des justiciables.

Le théâtre de John Day est le reflet d'un monde semé d'embûches pour celui qui ne sait pas se diriger dans le dédale des lois. On y voit des provinciaux un peu niais, comme par exemple le fïls d'un honnête hobereau du Norfolk qui vient s'encanailer et se faire plumer dans la capitale. Ailleurs, c'est la pauvre Bess qui est placée sous tutelle et qu'on veut dépouiller, ce ne sont partout qu'affaires d'héritages, de legs, d'hypothèques, de confiscation de biens meubles et immeubles, tout cela entraînant des complications juridiques inextricables. La justice et ses agents sont dangereux et malheur à celui qui ne sait pas ruser avec la loi, seule la voix des anges - c'est-à-dire celle des angelots sonnants et trébuchants - ou les charmes d'une femme peuvent faire avancer un procès et faire pencher le plateau de la balance dans un sens favorable. En effet le plaideur devait obtenir un ordre du roi pour que son cas soit jugé par la Cour de Westminster qui examinait moins le fond que la régularité de la procé- 
dure. Les pauvres, les soldats, les écrivains n'osent même plus aller à la Chambre Haute. L'innocence n'est pas suffisante pour être à l'abri des foudres de la justice. Un coup de rapière malheureux, une dette, de faux témoignages, tout peut conduire à la potence, au bannissement, à l'indigence, au mieux à la prison. Encore faut-il pouvoir payer sa pension au geôlier, sinon le prisonnier risque de moisir dans son cachot et devra se contenter de quelque brouet saumâtre $^{7}$. Délits politiques, délits de droit commun, il n'y a pas de distinction dans cette justice de théâtre plus vraie que nature. L'état de gentilhomme ne permet pas d'échapper à la prison, aux ceps, aux fourches patibulaires. Que l'on soit honnête ou non, coupable ou innocent, la justice apparaît plus comme un danger que comme une protection. Dans un tel univers, escrocs, roués et voleurs sont alors mieux préparés ou plus philosophes que les gens honorables.

Au-delà du soutien structurel et thématique, c'est surtout en homme de théâtre que John Day considère la justice. En effet la prison est un moyen pratique de faire disparaittre un personnage devenu gênant. En outre la justice aveugle et expéditive accélère et concentre l'intérêt dramatique, termes et sessions d'assises rythment une action par ailleurs défaillante. Le dramaturge connaît les goûts de son public et n'a d'autre désir que d'obtenir des applaudissements nourris, aussi n'épargnera-t-il pas les détails spectaculaires des exécutions ni les scènes grandguignolesques de torture. L'horreur par ses excès devient burlesque, Halibeck, le traitre persan dans The Travels est ainsi condamné à avoir successivement la langue arrachée, puis les mains coupées et enfin la tête tranchée ${ }^{8}$. D'une manière moins sensationnelle la jargon juridique apparaît comme un médium qui trouve dans le public un écho privilégié, esquisse les types et assure le comique. Les gens de loi sont experts dans le maniement de leur galimatias boursouflé de latin. 11 y a une telle abondance de mots qu'on pourrait en faire un lexique. Ces mots incompréhensibles, détournés de leur sens, sont de la parodie au premier degré, et au théâtre ils font mouche. Pour plagier l'adage qui veut que la fonction fasse l'homme, on peut dire qu'en ce qui concerne les hommes de loi, le langage fait la fonction. Enlevez à Lurdo, à Manasses -.. l'homme âne --, à Dametas leur jargon et il ne reste rien. Quand ils parlent ils trahissent doublement leur charge, car non 
seulement ils torturent le droit à leur profit mais ils l'interprètent et ne se contentent pas de le «dire», comme le prescrit Francis Bacon : «les juges doivent se rappeler que leur fonction est Ius dicere, et non pas Ius dare» ${ }^{9}$. Ainsi John Day laisse entendre que la justice n'est que l'art et l'artifice des mots qui composent les lois, qu'elle est l'expression de la procédure et non l'expression de ce qui est juste.

Il est évident que l'image que donne John Day de la justice, des juges et des prisons est aussi partielle que partiale, le dramaturge ne retient que les automatismes et le miroir se veut déformant. Mais la justice que nous avons vue jusqu'ici traitée comme sujet de parodie peut aussi devenir objet. Pour illustrer cette vision parallèle, on peut comparer des drames comme Gorboduc ou King Lear à The lle of Guls. Dans les trois cas il s'agit d'un prince qui désire léguer son pouvoir et ses biens à ses enfants; c'est au fond un très sérieux problème de droit. Or dans The Ile, le problème est posé d'emblée sur le plan ludique : Basilius lance un défi à ceux qui sauront séduire ses filles, premier enjeu, et la couronne, le sceptre, la main de justice seront le cadeau offert en prime aux vainqueurs. Ainsi la fonction de roi et ses attributs sont ridiculisés. Il est vrai que Day s'inspire largement de The Arcadia de Sidney qui s'achève sur un interminable procès qu'on peut aussi considérer comme une parodie. Parodie de parodie, le jeu se multiplie à l'infini. Un autre exemple montre comment le duel judiciaire, si solennel, si tragique, si déterminant dans Richard $I I$, vire franchement à la farce dans The Blind Beggar. Seuls le rituel, le cérémonial, le décorum sont respectés : défi, choix des armes, serment sur la Bible, rien n'est oublié mais tout devient grotesque. Mowbray et Bolingbroke ont cédé la place à de curieux combattants; barons, hobereaux et filous croisent le fer. Malgré la présence du roi, l'affrontement s'achève sur le spectacle de Strowd et de Momford qui se mesurent dans un jeu ridicule, à qui aura le plus d'angelots dans son gousset. Il y a donc transposition des valeurs les plus respectées, perversion de la notion de justice, tout devient objet de dérision. La pyramide de l'ordre traditionnel se présente bien souvent la pointe en bas, et le jeu des déguisements, autre forme de parodie, concourt à inverser les hiérarchies. La parodie est plus légère quand l'univers de 
la justice est un jeu de Colin Maillard, quand le vénérable juge Hortensio, dans Humour out of Breath, a les yeux bandés, comme l'image de la justice d'ailleurs, quand la rançon est un simple gage et quand un baiser peut délivrer le prisonnier ${ }^{10}$. Dans tout son théâtre, John Day s'ingénie à nous montrer un monde inversé, et, face aux gens de loi véreux, aux juges stupides, aux princes fantaisistes, il n'y a que des malandrins et des escrocs qui, par leur esprit, monopolisent la sympathie. C'est bien l'esprit (wit) qui les sauve du mépris et qui est érigé en une nouvelle et véritable institution, c'est là l'autre face de la justice. Ce double visage se retrouve dans The Alchemist de Ben Jonson, où Face entraîne la complicité du spectateur et gagne le pardon de son maître Lovewit. On n'éprouve même pas de pitié pour les victimes dans la mesure où elles sont d'abord des dupes, victimes d'elles-mêmes, privées un moment de bon sens, le bon sens qui est le premier état de la justice.

Si la justice en tant qu'institution reste, du moins en apparence, à l'abri de la parodie, John Day n'épargne pas ses agents; dans la peinture qu'il en fait, le trait est grossi, noirci jusqu'à la caricature. Comme dans Les Guêpes d'Aristophane, chacun rêve de se faire construire un petit tribunal devant sa porte, chacun fait sa justice, chacun est la justice. Lurdo, semblable au caméléon, prend les couleurs de la loi, c'est la loi qui s'exprime par sa bouche, il est la loi. Rien de très étonnant quand on sait qu'en anglais le mot «justice» désigne à la fois la justice et le juge. La noirceur de son caractère est soulignée par les étapes de sa carrière : usurier, faussaire, délateur. Son aveu est révélateur : «j’ai pigeonné l'univers tout entier», proclame-t-il. Le monde feutré,fourré des gens de loi, qui sont souvent de respectable souche provinciale, est démystifié sans ménagement. Manasses par exemple, évoque son grand-père chasseur de rats et son père bourreau. Lui-même, indicateur de profession, a réussi à s'acheter chez le frippier une robe pour pouvoir plaider. Ainsi, avec un habit, avec un peu de latin et heaucoup de ruse, en voilà assez pour faire un authentique vice-procureur au service de Dametas, autre détestable entremetteur de la justice. Ce dernier, intermédiaire indispensable, fourbe et vénal, rêve d'une justice démente dont il serait l'ordonnateur, il se plaît à imaginer qu'il pourrait obtenir un privilège, une patente qui lui permettrait de pendre à sa guise $24 \mathrm{co-}$ 
quins, 6 chevaliers, 10 bouffons, 13 félons et 14 traitres par an $^{11}$. Avec Dametas, l'homme de loi n'est que la caricature d'un sinistre bouffon, mais le bouffon n'est-il pas souvent le révélateur des vices de la société ? Le vice ou la vertu ne se révèle qu'en situation, et l'amalgame qui se fait entre le caractère grotesque de l'individu et sa fonction, jette le discrédit sur ce qu'il représente. La'parodie du juge rejaillit nécessairement sur la justice.

Pour conclure on peut dire que c'est en dramaturge que John Day aborde le problème de la justice. Le mythe n'étant plus assez puissant pour cristalliser l'intérêt des spectateurs, c'est des gens de loi, des escrocs et des dupes que se nourrit son théâtre. Le moraliste reste discret, et au fond très conventionnel, la subversion est de surface, et même si «des sottises du temps il compose son fiel», ses parodies restent en deça de la satire.Le jeune prince Polymetes dans Law Tricks, déclare solennellement que la loi est sacrée, il la compare à une chaîne d'or qui lie entre eux ceux qui composent la république. La loi est sœur jumelle de la justice, elle protège les pauvres et quant aux magistrats ils doivent être les ministres de cette justice divine. Cette notion de justice et d'équité, garante de l'équilibre social et économique, est développée dans The Parliament of Bees où la sagesse et l'humour adoucissent le ricanement cynique du satiriste désabusé. Loin de saper les fondements d'un ordre chancelant et contesté au tournant du siècle, John Day montre un esprit critique, dénonce impostures et imposteurs mais reste assez lucide et possède assez de bon sens pour savoir que la justice ne peut régner qu'au pays d'Obéron.

Jean-Pierre VILLQUIN 


\section{NOTES}

1. L'édition de référence est celle de M. Robin Jeffs, The Works of John Day, Holland Press, (London, 1963).

2. Iusticia, plume à l'encre brune, Bruegel 1559. Bruxelles, Bibliothèque Royale Albert I; Inv. no S II 133707.

3. Boy. My Lord, your keeper hath brought a lady or two to see you. Asp. To see me ? why, am I turnd monster? doth he take money to shew me? what doth a take a peece, troe ?

Humour out of Breath, p. 462.

4. Can. Well, Mr. Strowd, as I was passing though Allgate this morning I saw the Shreeves and Constables set towards to Newgate to fetch your father : the Carpenter in a Cart carried the Jebbet to Bednall-Green.

The Blind Beggar, p. 56.

5. Voir Peregrinatio Scholastica, «The sevententhe tractate», pp.517519.

6. Humour, pp. 417-418.

7. Iay. According to my warrant heere I must this morning fetch my prisoner to ayring. Hee had need bee hung least his flesh should mould, for I am sure his clothes are mustie already ... these fiue or six moneths at least he has had nothing but the hard boords for his bed, drie bread for his foode and miserable water for his drinke...

The Travels, pp. 389-390.

Ailleurs, Assistance déclare à Aspero : «Iwarrant you, my Lord, let me alone, and we knew not how to abuse a prisoner, we were not worthy to be a iaylor. Humour, p. 466.

8. The Travels, p. 401

9. Bacon, Essays, LVI, Of Iudic ature.

10. Humour, pp. 462-464.

Il y a dans la même pièce une aimable parodie quand Florimell retient son amant prisonnier dans sa chambre (voir p. 443).

11. The Ile of Guls, p. 224. 